\title{
Inactivation of urease by catechol: kinetics and structure
}

Luca Mazzei $^{\mathrm{a}}$, Michele Cianci ${ }^{\mathrm{b}}$, Francesco Musiani ${ }^{\mathrm{a}}$, Gábor Lente ${ }^{\mathrm{c}}$, Marta Palombo $^{\mathrm{a}}$, Stefano Ciurli ${ }^{\text {a,* }}$

a Laboratory of Bioinorganic Chemistry, Department of Pharmacy and Biotechnology, University of Bologna, Italy.

${ }^{\mathrm{b}}$ European Molecular Biology Laboratory, DESY, Hamburg, Germany.

${ }^{\mathrm{c}}$ Department of Inorganic and Analytical Chemistry, University of Debrecen, Hungary.

Corresponding author: Stefano Ciurli, Laboratory of Bioinorganic Chemistry, Department of Pharmacy and Biotechnology, University of Bologna, Viale G. Fanin 40, I-40127, Bologna, Italy. E-mail: stefano.ciurli@unibo.it

Keywords: urease inhibition, catechol, nickel, kinetic measurements, X-ray crystallography. 


\begin{abstract}
Urease is a Ni(II)-containing enzyme that catalyzes the hydrolysis of urea to yield ammonia and carbamate at a rate $10^{15}$ times higher than the uncatalyzed reaction. Urease is a virulence factor of several human pathogens, in addition to decreasing the efficiency of soil organic nitrogen fertilization. Therefore, efficient urease inhibitors are actively sought. In this study, we describe a molecular characterization of the interaction between urease from Sporosarcina pasteurii (SPU) and Canavalia ensiformis (jack bean, JBU) with catechol, a model polyphenol. In particular, catechol irreversibly inactivates both SPU and JBU with a complex radical-based autocatalytic multistep mechanism. The crystal structure of the SPU-catechol complex, determined at $1.50 \AA$ resolution, reveals the structural details of the enzyme inhibition.
\end{abstract}

\title{
1. INTRODUCTION
}

Urease is a non-redox metallo-enzyme involved in the global nitrogen cycle in plants, algae, fungi, and several microorganisms [1, 2]. Its fully conserved bimetallic Ni(II)-containing reaction site (Fig. 1A) catalyzes the rapid hydrolytic decomposition of urea to give ammonia and carbamate $[1,2]$. The uncatalyzed hydrolysis of these reaction products causes an overall $\mathrm{pH}$ increase that has negative implications for human health [3] as well as for the environmental ecosphere [4]. In particular, urease plays an essential role in the virulence of the human pathogen Helicobacter pylori. This bacterium is able to use the urease activity to create a local $\mathrm{pH}$ environment compatible with its survival in the acidic conditions of the gastric mucosa [5]. It has been estimated that approximately two-thirds of the world's population harbor $H$. pylori [6]. This microorganism has been classified, uniquely among bacteria, as a class-I carcinogen in humans [7]. As a consequence of these facts, urease is the ideal target for the treatment of diseases caused by a large variety of ureolytic human pathogens in addition to H. pylori, such as Cryptococcus neoformans, Yersinia enterocolitica, 
Proteus mirabilis, and Mycobacterium tuberculosis [8-11]. The urease inhibitors characterized so far [12] can be roughly divided in two main classes, based on the mode of interaction with the enzyme. The first class contains molecules (phosphorodiamidate, phosphate, thiols, sulfite, fluoride as well as hydroxamic, citric and boric acids) directly binding to the $\mathrm{Ni}(\mathrm{II})$ ions in the active site $[1,2,13,14]$. The second class of inhibitors is composed of molecules such as 2-mercapto-ethanol (BME) [15] and 1,4-benzoquinone (PBQ) [16] that have been proven to bind to the $\mathrm{S} \gamma$ atom of the conserved $\alpha \mathrm{Cys} 322$ [Sporosarcina pasteurii urease (SPU) numbering]. This residue is located onto a mobile helix-turn-helix motif (flap) that is essential for catalysis by modulating the transit of substrate and products through the active site cavity (Fig. 1B). BME can actually be ascribed to both classes, because it is able to further use its thiol moiety to coordinatively bridge the $\mathrm{Ni}$ (II) ions in the active site [15]. On the other hand, PBQ is a representative of the most efficient class of urease inhibitors characterized so far [17]. The structure of SPU inactivated by PBQ shows that the thiol group of $\alpha$ Cys322 forms a covalent adduct with PBQ, blocking enzyme activity by rendering the flap unable to move [16]. A similar inactivation mode could potentially represent the basis for the inhibitory activity of polyphenols toward urease. These molecules comprise a wide class of natural compounds found in fruits, vegetables, cereals, tea, coffee and wine, and are generally recognized as beneficial to human health for their antioxidant properties [18]. This class of urease inhibitors has also been recently described as efficient against $H$. pylori, but their mode of action has not been understood so far [19-22]. Here we present the kinetic and structural characterization of SPU and Canavalia ensiformis (jack bean, JBU) inactivation by catechol (benzene-1,2-diol, CAT), the simplest molecule featuring a polyphenol scaffold that was identified as the most promising inhibitor of soil urease [17]. 


\section{MATERIALS AND METHODS}

\subsection{Enzymes.}

Sporosarcina pasteurii urease was expressed and purified from the native source, following a previously described protocol $[14,16]$. The protein was quantified by measuring the activity and considering its specific activity of 2500 units $\mathrm{mg}^{-1}$ and $\mathrm{M}_{\mathrm{r}}=250 \mathrm{kDa}$. Jack bean urease type C-3, powder ( $\geq 600,000$ units/g) was purchased from Sigma-Aldrich and was quantified following manufacturer's information.

\subsection{Kinetic measurements.}

Pre-incubation experiments were carried out at room temperature by using a spectrophotometric assay in which cresol red is exploited as a probe to follow the overtime increase of $\mathrm{pH}$ due to urease activity, following a protocol previously described [16]. In the case of SPU - catechol experiments, a solution of $50 \mathrm{nM}$ SPU dissolved in 2 mM HEPES buffer, $\mathrm{pH} 7.5,1 \mathrm{mM} \mathrm{Na} \mathrm{SO}_{3}$, was diluted down to $1 \mathrm{nM}$ final concentration in the cresol red reaction solution, containing $30 \mathrm{mg} \mathrm{L}^{-1}$ cresol red in $2 \mathrm{mM}$ HEPES buffer, $\mathrm{pH} 7.5,2 \mathrm{mM}$ EDTA. By doing this, $\mathrm{Na}_{2} \mathrm{SO}_{3}$ was diluted 50 -fold as well, down to $20 \mu \mathrm{M}$ final concentration. In the case of JBU - catechol experiments, a solution of $250 \mathrm{nM} \mathrm{JBU}$ dissolved in $2 \mathrm{mM}$ HEPES buffer, $\mathrm{pH} 7.5$, without $\mathrm{Na}_{2} \mathrm{SO}_{3}$, was directly diluted to $2.5 \mathrm{nM}$ in the same cresol red solution. Then, different concentrations of catechol solutions (4-16 mM and 5-40 $\mu \mathrm{M}$ for the experiments on SPU and JBU, respectively) dissolved in $2 \mathrm{mM}$ HEPES buffer, $\mathrm{pH}$ 7.5 (containing $20 \mu \mathrm{M} \mathrm{Na}_{2} \mathrm{SO}_{3}$ in the case of experiments on SPU) were added, taking the time when the enzyme solution and the ligand are mixed as zero time of incubation. After appropriate periods of time, aliquots were withdrawn from the incubation solution, $100 \mathrm{mM}$ urea was added, and the change in absorbance over time was monitored $(\lambda=573 \mathrm{~nm})$. The 
activity was calculated by a linear fitting of the straight portion in the absorbance vs. time curve and normalized to the activity measured at time zero incubation.

\subsection{Crystallization, data collection and structural determination.}

A solution of Sporosarcina pasteurii urease (SPU, $1 \mathrm{mg} \mathrm{mL}^{-1}$ ) in $20 \mathrm{mM}$ HEPES buffer, $\mathrm{pH}$ 7.5, was incubated in the presence of $1 \mathrm{mM}$ catechol (dissolved in the same buffer) for $2 \mathrm{~h}$ and then concentrated to $11 \mathrm{mg} \mathrm{mL}^{-1}$. Subsequently, $2 \mu \mathrm{L}$ of this solution was diluted with 2 $\mu \mathrm{L}$ of the precipitant solution (1.6-2.0 M ammonium sulfate in a $50 \mathrm{mM}$ sodium citrate buffer $\mathrm{pH}$ 6.3). Crystallization was performed at $293 \mathrm{~K}$ using the hanging-drop method, equilibrating the drop against $0.5 \mathrm{~mL}$ of the precipitant solution using Quiagen EasyXtal 15-Well plates. Rice-shaped protein crystals appeared in 1-2 weeks and grew to a size of $0.1 \times 0.1 \times 0.3 \mathrm{~mm}^{3}$. Crystals were scooped up using cryoloops, transferred to a cryoprotectant solution containing $20 \%$ ethylene glycol, $2.4 \mathrm{M}$ ammonium sulfate in a $50 \mathrm{mM}$ sodium citrate buffer at $\mathrm{pH} 6.3$ and then flash-cooled and stored in liquid nitrogen. Diffraction data were collected at $100 \mathrm{~K}$ using synchrotron radiation at the EMBL P13 beamline of the Petra III storage ring, c/o DESY, Hamburg (Germany). Diffraction images were collected by performing helical scans along the crystal to achieve higher resolution by minimizing radiation damage. Data were processed using XDS [23] and AIMLESS [24]. The crystals were isomorphous with those of native urease and other complexes of the same enzyme. The model of SPU with its highest resolution available so far, that is in complex with citrate (PDB code 4AC7, 1.50 A resolution [25]) and devoid of water molecules and ligands, was used as a starting model for the rigid body refinement of the single $\alpha \beta \gamma$ protein trimer, carried out using Refmac [26]. Model building and water or ligand addition/inspection were conducted using Coot [27]. The structure was refined using isotropic atomic displacement parameters (ADPs), including the hydrogen atoms in the riding positions, and then refined using anisotropic ADPs. The model was then validated using the PDB_REDO web server [28]. The final $\mathrm{R}$ and $\mathrm{R}_{\text {free }}$ were 12.12 
and 15.42, respectively. The diffraction data and final refinement statistics are given in Table S2. The structure was deposited in the Protein Data Bank under the accession code 5G4H. Figures were generated using UCSF Chimera [29], PyMol [30], and CrystalMaker (http://www.crystalmaker.com).

\subsection{Quantum mechanical calculations.}

Density functional theory (DFT) computations were carried out using the program ORCA 3.0.3 [31] and the Becke three-parameter hybrid functional combined with Lee-Yang-Parr correlation functional (B3LYP/G) $[32,33]$ as defined in the Gaussian software [34]. The formation of the bond between the $\mathrm{C} 3$ atom of ortho-benzoquinone (cyclohexa-3,5-diene-1,2dione, OBQ) or ortho-semiquinone [(2-hydroxyphenyl)-oxidanyl, OSQ] and $\alpha \mathrm{Cys} 322(\mathrm{~S} \gamma)$ was investigated using relaxed scan computations [31], which involve constrained optimizations for different values of a selected reaction coordinate. The chosen coordinate, in this case the bond distance, is fixed to a certain value while the remaining coordinates of the molecule are optimized. After completion of one optimization cycle, the value of the reaction coordinate is changed and another optimization cycle is carried out. This procedure was used to trace a path (theoretically close to a minimum-energy path) from the separated OBQ (or OSQ) and a molecule of methanethiol, used to model $\alpha$ Cys322 thiol, to the product. For relaxed scan calculations all atoms were described by the Pople-style 6-311G(d,p) [35] basis set. The Dunning correlation-consistent polarized double zeta basis set (cc-pVDZ) [36] was used to calculate the hydrogen bond network around the catechol moiety bound to the mobile flap of SPU, while the Dunning correlation-consistent polarized triple zeta basis set, with the inclusion of diffuse functions (aug-cc-pVTZ) [36], was used for computations on small molecules. Frequency computations were executed on the molecules (reported in Fig. S3 and Table S5) as well as on the critical points reported in Fig. 4, in order to determine their nature. 


\section{RESULTS AND DISCUSSION}

The inhibition of Sporosarcina pasteurii urease (purified according to previously published protocols $[14,16])$ by catechol was studied by pre-incubating the enzyme with different concentrations of inhibitor for increasing periods of time in the absence of substrate, and the residual activity was monitored using a spectrophotometric assay [16]. The data in Fig. 2A show the presence of an initial lag phase, whose extent decreases as the concentration of catechol increases, followed by an acceleration that eventually yields the complete inhibition of the enzyme. This behavior is typical for irreversible enzyme inactivators, known to form stable covalent adducts between the inhibitor and essential functional groups of the enzyme [37].

The observed kinetic response suggests the presence of a process that converts catechol, inactive at the start of the reaction, into another compound, which then acts as the actual inactivator of the enzyme. Catechol is known to undergo oxidation in neutral aqueous solutions upon exposure to air [38]. The need for dissolved molecular oxygen was confirmed by the essential lack of SPU inactivation when the kinetic experiments described above were performed in controlled oxygen-free atmosphere (Fig. S1 in the Supplementary Material). According to the known reduction potentials (see Table S1), the formation of the orthosemiquinone radical or of ortho-benzoquinone are thermodynamically favorable processes, as is the disproportionation of the ortho-semiquinone to form ortho-benzoquinone and catechol. These reactions are expected to be much faster than the observed lag phase [39], which therefore cannot be ascribed to these processes. We thus investigated the possibility that sulfite, needed in the assay solution to stabilize SPU activity [14], could quench the urease inactivation by acting as a reducing agent of either OBQ or OSQ, or both, as suggested by the reduction potentials shown in Table S1. In addition, sulfite can also react with quinones, forming dihydroxybenzene-sulphonates shown to be inactive towards the cysteine thiols of SPU [16]. In order to explore this hypothesis, the same kinetic experiments were conducted 
using, in place of SPU, Canavalia ensiformis urease (jack bean urease, JBU), which does not require the presence of sulfite as a preservative. Fig. $2 \mathrm{~B}$ shows that, in this latter case, the initial lag phase is almost completely abolished, supporting the role of sulfite in slowing down the inactivation of SPU. In addition, the concentration of catechol needed to exert similar effects on urease activity is three orders of magnitude smaller in the case of JBU as compared to that needed for SPU. The sigmoidal shape of the JBU inactivation data as a function of incubation time suggests the occurrence of a collectively autocatalytic process, namely a set of chemical reactions that yield, as reaction products, one or more catalysts that accelerate the inhibition of JBU [40]. The nature of this autocatalytic process for JBU inactivation must involve the production of derivatives of catechol generated by reaction with dioxygen, namely OSQ and/or OBQ. We speculate here that a similar autocatalytic process also occurs for SPU, but only upon consumption of sulfite, when the fast phase of SPU inactivation is initiated by unrestrained formation of the oxidation products of catechol.

The derivation of a complete kinetic model able to interpret the data requires the identification of the reacting species and of the reaction product(s). Previous studies on the inactivation of JBU by catechol were interpreted with the covalent modification of $\alpha$ Cys592 (corresponding to $\alpha$ Cys322 in SPU) through a Michael-type nucleophilic addition by its thiol group on OBQ, even though a radical-based mechanism was not ruled out [41]. In the most general case, this residue could react with either OSQ or OBQ, depending on the nature of the reaction mechanism: a nucleophilic Michael-type addition on OBQ or a radical addition on OSQ. Furthermore, two different products could be a priori obtained, depending on the formation of a S-C covalent bond in ortho (C3 carbon) or in meta (C4 carbon) with respect to the hydroxyl groups on the aromatic ring. In order to understand the structural basis of urease inactivation by catechol, a crystallographic structural study of the products of inactivation of SPU by this inhibitor was carried out. 
SPU was incubated with catechol for two hours before crystallization. The resulting crystals were amenable to X-ray diffraction analysis, yielding a $1.50 \AA$ resolution structure (see Table S2 for the full details of X-ray data collection, processing and analysis). The structure confirms the well-known molecular architecture of SPU together with the rigidity of the protein scaffold (root mean square deviation for the $\mathrm{C} \alpha$ residue atoms $=0.08 \AA$ with respect to the structure of native urease; PDB code 4CEU). The coordination environment of $\mathrm{Ni}(\mathrm{II})$ is almost identical to that observed in the case of native SPU (Table 1 and Table S3). The mobile flap covering the active site cavity is found in the open conformation, as also found in all SPU structures determined so far, with the distinguished exception of the complex with diamidophosphate (DAP), an analogue of the intermediate or transition state of the reaction [42].

The unbiased omit electron density map was calculated with Fourier coefficients $F_{o}-F_{c}$ and phases derived from the refinement of SPU-catechol structure using the citrate-inhibited SPU (PDB code 4AC7, $1.50 \AA$ resolution [25]) as a starting model after removal of the citrate moiety and other water molecules/ligands present in the 4AC7 model. This omit map showed an additional electron density in the vicinity of the flap and proximal to the $\alpha \mathrm{Cys} 322$ residue (Fig. S2), which was successfully modeled with full occupancy using the aromatic dihydroxylated moiety of the inhibitor (Fig. 3A). The final refined structure features a covalent bond between $\alpha \mathrm{Cys} 322 \mathrm{~S} \gamma$ and the aromatic $\mathrm{C} 3$ atom of the inhibitor, with a C-S distance of $1.65 \AA$ (Fig. 3B and Table S4). The dihydroxylated aromatic moiety points towards the Ni(II) ions. The solvent-exposed $\alpha$ Cys555, far from the active site, is not modified by catechol, as observed in the case of PBQ bound SPU [16], emphasizing the peculiarity of the environment around $\alpha \mathrm{Cys} 322$. This feature could be related to the presence of the $\alpha$ His323 residue in the adjacent position, which is conserved and known to be essential for urease activity. A third cysteine residue ( $\alpha \mathrm{Cys} 520)$ is protected from the solvent and is not affected by any modification. 
The oxidation state of the group bound to $\alpha \mathrm{Cys} 322 \mathrm{~S} \gamma$ (reduced catechol or oxidized orthobenzoquinone) was investigated using quantum-mechanical calculations carried out on these two possible redox moieties (see Supplementary Material for details). The results suggest the presence of the reduced form, and the final crystallographic refinement was thus carried out accordingly. In order to assess the position of the hydrogen atoms around the catechol moiety covalently bound to $\alpha \mathrm{Cys} 322 \mathrm{~S} \gamma$, attention was focused on the network of H-bonds involving this modified residue and the water molecules in its proximity (see Supplementary Material for details). In particular, $\mathrm{W}_{\mathrm{A}}$ and $\mathrm{W}_{\mathrm{B}}$ are bound to CAT O1 with O-O distances of 2.52 and $3.05 \AA$, respectively, while $\mathrm{W}_{\mathrm{C}}$ is at $\mathrm{H}$-bond distance from both CAT O1 and O2 (at 3.14 and $2.54 \AA$, respectively). $\mathrm{W}_{\mathrm{A}}$ also forms two H-bonds with the backbone O atoms of $\alpha$ Lys 169 and $\alpha$ Leu365. $\mathrm{W}_{\mathrm{C}}$ is at $\mathrm{H}$-bond distance from both $\alpha \mathrm{Ala} 366 \mathrm{O}$ and $\mathrm{W}_{\mathrm{G}}$, and the latter is $\mathrm{H}$ bonded to the sulfate ion located next to the Ni(II) ions. Quanto-mechanical optimization of the H-bonding network between the catechol moiety, the surrounding residues and water molecules suggest that $\mathrm{W}_{\mathrm{A}}$ is actually the hydronium $\mathrm{H}_{3} \mathrm{O}^{+}$ion; this induces the $\mathrm{H}$ atoms of the hydroxyl groups of catechol to lie on the same plane of the aromatic ring, forming an intra-molecular H-bond between a $\alpha \mathrm{Cys} 322 \mathrm{~S} \gamma \mathrm{p}$-orbital and the $\mathrm{O}-\mathrm{H}$ group bound to the $\mathrm{C} 2$ atom of catechol [43].

The present crystal structure of SPU inactivated by catechol, with the $\alpha \mathrm{Cys} 322$ thiol bound to the $\mathrm{C} 3$ atom of the aromatic ring, rules out the formation of the meta adduct (cysteine thiol bound to the $\mathrm{C} 4$ atom of catechol). In order to distinguish between the nucleophilic attack on OBQ and the radical-based reaction mechanism involving OSQ, quanto-mechanical calculations were carried out (see Supplementary Material for details). In both cases, the reaction involves the formation of a bond between $\mathrm{OBQ} / \mathrm{OSQ}(\mathrm{C} 3)$ and $\alpha \mathrm{Cys} 322(\mathrm{~S} \gamma)$. The first mechanism taken into account was the nucleophilic attack performed by the methanethiol (MeSH) sulfur atom to the $\mathrm{C} 3$ atom of OBQ. The relaxed surface scan for this reaction coordinate leads to a gradual energy increase as the $\mathrm{OBQ}(\mathrm{C} 3)-\mathrm{MeSH}(\mathrm{S})$ distance is shortened, 
without the formation of any critical point on the potential energy surface (Fig. S5). This result is somehow expected, considering that the $\mathrm{C} 3$ atom of OBQ has a slightly negative partial charge (Table S5), not compatible with a nucleophilic attack. We then took into account the direct interaction between $\mathrm{OSQ}(\mathrm{C} 3)$ and $\mathrm{MeSH}(\mathrm{S})$ (blue pathway in Fig. 4). The surface scan along the $\mathrm{OSQ}(\mathrm{C} 3)-\mathrm{MeSH}(\mathrm{S})$ coordinate resulted in a barrier of ca. $39 \mathrm{kcal} / \mathrm{mol}$ and the identification of a transition state (TS1) characterized by $\mathrm{C} 3$ in a distorted tetrahedral geometry, together with a larger MeSH S-H bond distance as compared to that found in MeSH. Along this pathway, TS1 then evolves to a radical reaction intermediate (INT1) characterized again by $\mathrm{C} 3$ in a distorted tetrahedral geometry and full protonation of the two hydroxyl groups on the aromatic ring. The detailed analysis of the molecular orbitals of OSQ subsequently showed that the largest fraction of the spin density is localized on the $\mathrm{O} 2$ atom (Table S5), suggesting that the reaction could actually occur in two steps (Scheme 1), as previously suggested $[44,45]: i)$ in the first step the thiol $\mathrm{H}\left(\mathrm{H}_{\mathrm{S}}\right)$ is taken up by the $\mathrm{O} 2$ of OSQ to form a molecule of catechol and a methylsulfanyl radical $\left(\mathrm{MeS}^{\bullet}\right)$, and $i$ ) the $\mathrm{S}$ atom of $\mathrm{MeS}^{\bullet}$ then attacks the $\mathrm{C} 3$ of catechol to give the reaction products (red pathway in Fig. 4). The exploration of the $\mathrm{OSQ}(\mathrm{O} 2)-\mathrm{MeSH}\left(\mathrm{H}_{\mathrm{S}}\right)$ reaction coordinate (Fig. 4) revealed a first energy maximum located at ca. $14 \mathrm{kcal} / \mathrm{mol}$ higher than the initial molecules, leading to the identification of the transition state TS2 characterized by the MeSH hydrogen already bound to OSQ $\mathrm{O} 2$ atom. TS2 then evolves to yield catechol and the $\mathrm{MeS}^{\bullet}$ radical. The exploration of the $\mathrm{CAT}(\mathrm{C} 3)-\mathrm{MeS}^{\bullet}(\mathrm{S})$ reaction coordinate then leads to a second energy maximum characterized as the transition state (TS3) located at ca. $9.5 \mathrm{kcal} / \mathrm{mol}$ higher than the adduct formed by catechol and the $\mathrm{MeS}^{\bullet}$ radical. TS3 features the $\mathrm{C} 3$ atom of catechol in a distorted tetrahedral geometry. TS3 then evolves into the same radical reaction intermediate INT1 observed in the reaction pathway initially explored, with very similar geometry and energy with respect to TS3. In both pathways, INT1 can be eventually stabilized by a third molecule able to extract a radical $\mathrm{H}$ atom and give the reaction product observed in the crystal structure 
of catechol-bound SPU. This third player could be either molecular oxygen or another molecule of OSQ (to give the fully reduced catechol-like moiety), or OBQ (to give OSQ). These calculations, coupled with the structural data, strongly support the involvement of the ortho-semiquinone radical in the reaction with the active form of urease to give the inactive enzyme.

The working hypothesis for urease inactivation by catechol thus implicates the set of reactions portrayed in Scheme 2. The OSQ radical can be generated by oxidation of catechol by dissolved $\mathrm{O}_{2}$ through the formation of superoxide (reaction 1) and by comproportionation of catechol and ortho-benzoquinone (reaction 2). The active form of urease $\left(\mathrm{JBU}_{\text {act }}\right.$ in the case of JBU) then reacts with OSQ to give the inactive enzyme (JBU $\mathrm{inh}_{\text {nh }}$ according to the reaction mechanism based on quanto-mechanical calculations, as described above (reaction 3). The final reaction 4 takes into account a step that produces ortho-benzoquinone ${ }^{\mathrm{a}}$. This model involves a number of variables, one of them being the concentration of dissolved oxygen $\left(\left[\mathrm{O}_{2}\right]\right)$. Considering that the experiments were carried out using vessels always open to air, and given the relatively long time scale of the enzyme inactivation (thousands of seconds), it is assumed that the oxygen concentration is constant during the process and equal to the initial air-saturated value $\left[\mathrm{O}_{2}\right]_{0}$ at the temperature of the experiments, i.e. $\left[\mathrm{O}_{2}\right] \approx\left[\mathrm{O}_{2}\right]_{0}=$ $0.2 \mathrm{mM}$ [46]. The following, reasonably simple analytical expression can be derived (see the Supplementary Material for the details of the mathematical treatment) [47] to give a good fit to the detected curves [Eq. (1)].

$$
\left[\mathrm{JBU}_{\mathrm{act}}\right]=\left[\mathrm{JBU}_{\mathrm{act}}\right]_{0} \exp \left(-k_{a p p 1} t-k_{a p p 2} t^{2}\right)
$$

\footnotetext{
${ }^{\text {a }}$ Superoxide is not a reactant in any of the considered reactions in Scheme 2; most probably it will undergo dismutation to generate dioxygen and hydrogen peroxide, but this reaction does not influence the kinetic signature analyzed here.
} 
In this equation, $k_{a p p l}$ and $k_{a p p 2}$ are functions of the kinetic constants $k_{1}, k_{2}, k_{3}$ and $k_{4}$ in Scheme 2, as well as the initial concentration of catechol, [CAT] $]_{0}$, as indicated in Eq. (2) and Eq. (3), which were derived through solution of the system of simultaneous ordinary differential equations by applying the steady state approximation and the Taylor series comparison technique [48] (see Supplementary material for the full mathematical treatment):

$k_{a p p 1}=\frac{k_{1} k_{3}}{k_{4}}[\mathrm{CAT}]_{0}$

$k_{a p p 2}=\frac{2 k_{1} k_{2} k_{3}}{k_{4}}[\mathrm{CAT}]_{0}^{2}-\frac{k_{1}^{2} k_{3}}{k_{4}}\left[\mathrm{O}_{2}\right]_{0}[\mathrm{CAT}]_{0}$

A simultaneous fit of the data obtained for JBU at four different initial catechol concentrations using this model provided a very good agreement between theory and experiment (Fig. 2B), yielding the following values: $k_{1}=0.9 \pm 0.1 \mathrm{M}^{-1} \mathrm{~s}^{-1}, k_{2}=49 \pm 4 \mathrm{M}^{-1} \mathrm{~s}^{-1}$ and $k_{3} / k_{4}=29 \pm 4^{\mathrm{b}}$. The much larger value of $k_{2}$ as compared to $k_{1}$ is consistent with the observed autocatalytic process, while the $k_{3} / k_{4}$ value significantly larger than one implies that the reaction continues until complete consumption of the active enzyme.

\footnotetext{
${ }^{b}$ This model was not applicable to the case of SPU because the active role of sulfite would impose additional reactions, increasing the number of parameters to fit and decreasing their reliability; furthermore, the use of a much larger concentration of catechol to observe an inhibition effect, due to the presence of sulfite, together with the value of $k_{l}$ obtained in the case of JBU, implies that, in the case of SPU, the concentration of dioxygen in solution is no longer constant; these factors would complicate the model for SPU without adding any significant information on the intrinsic inhibition of urease by catechol.
} 


\section{CONCLUSIONS}

The molecular details of the urease irreversible inactivation by catechol have been elucidated. This molecule inactivates urease by a radical-based mechanism that yields a covalent adduct with the conserved $\alpha$ Cys322 located on the flap that modulates the opening and closing of the active site channel, blocking it in the open position (see Fig. S6). This seems a general trait, common to aromatic poly-hydroxylated urease inhibitors, as it has been observed recently also for the case of urease irreversible inactivation by para-benzoquinone [16]. The role of reducing agents such as sulfite on this reaction has also been clarified. Finally, a kinetic scheme of urease inactivation by catechol was elaborated and positively tested against experimental data. The obtainment of key structure-activity relationships for the development of improved poly-hydroxylated aromatic molecules as urease inactivators, with potential applications in medicine as well as in agro-environmental settings, is under active investigation in our laboratory.

\section{ABBREVIATIONS}

SPU Sporosarcina pasteurii urease

JBU Canavalia ensiformis (jack bean) urease

CAT catechol

OBQ ortho benzoquinone

OSQ ortho-semiquinone radical

PBQ 1,4-benzoquinone

BME 2-mercapto-ethanol

DAP diamidophosphate 


\section{ACKNOWLEDGMENTS}

We thank Dr Vara, from the Dept. of Pharmacy and Biotechnology of the University of Bologna, for his assistance in S. pasteurii cell growth. LM is a Ph.D. fellow supported by the University of Bologna; FM is a research assistant supported by CIRMMP (Consorzio Interuniversitario di Risonanze Magnetiche di Metallo-Proteine) and the University of Bologna; MP is supported by Specialty Fertilizer Products (Leawood, KS, USA). X-ray diffraction data were collected under the beam time award number MX-333 from the European Molecular Biology Laboratory (EMBL, Petra III, 399. c/o DESY, Hamburg, Germany).

\section{APPENDIX A. SUPPLEMENTARY DATA}

Supplementary data for catechol-bound SPU have been deposited at the Protein Data Bank with accession code 5G4H. Supplementary data associated with this article can be found in the online version.

\section{REFERENCES}

[1] B. Zambelli, F. Musiani, S. Benini, S. Ciurli, Chemistry of $\mathrm{Ni}^{2+}$ in urease: sensing, trafficking, and catalysis, Acc. Chem. Res. 44 (2011) 520-530.

[2] M.J. Maroney, S. Ciurli, Nonredox nickel enzymes, Chem. Rev. 114 (2014) 4206-4228.

[3] J.C. Rutherford, The emerging role of urease as a general microbial virulence factor, PLoS Pathog. 10 (2014) e1004062.

[4] S. Kiss, M. Simihaian, Improving efficiency of urea fertilizers by inhibition of soil urease activity, Kluwer Academic Publishers, Dordrecht, The Netherlands, 2002.

[5] J.L. Slonczewski, M. Fujisawa, M. Dopson, T.A. Krulwich, Cytoplasmic pH measurement and homeostasis in bacteria and archaea, Adv. Microb. Physiol. 55 (2009) 1-79, 317. 
[6] J.G. Kusters, A.H. van Vliet, E.J. Kuipers, Pathogenesis of Helicobacter pylori infection, Clin. Microbiol. Rev. 19 (2006) 449-490.

[7] Schistosomes, liver flukes and Helicobacter pylori. IARC Working Group on the Evaluation of Carcinogenic Risks to Humans. Lyon, 7-14 June 1994, IARC monographs on the evaluation of carcinogenic risks to humans / World Health Organization, International Agency for Research on Cancer 61 (1994) 1-241.

[8] W. Lin, V. Mathys, E.L. Ang, V.H. Koh, J.M. Martinez Gomez, M.L. Ang, S.Z. Zainul Rahim, M.P. Tan, K. Pethe, S. Alonso, Urease activity represents an alternative pathway for Mycobacterium tuberculosis nitrogen metabolism, Infect. Immun. 80 (2012) 2771-2779. [9] G.M. Young, D. Amid, V.L. Miller, A bifunctional urease enhances survival of pathogenic Yersinia enterocolitica and Morganella morganii at low pH, J. Bacteriol. 178 (1996) 6487-6495.

[10] G.M. Cox, J. Mukherjee, G.T. Cole, A. Casadevall, J.R. Perfect, Urease as a virulence factor in experimental cryptococcosis, Infect. Immun. 68 (2000) 443-448.

[11] B.D. Jones, C.V. Lockatell, D.E. Johnson, J.W. Warren, H.L.T. Mobley, Construction of a urease-negative mutant of Proteus mirabilis: analysis of virulence in a mouse model of ascending urinary tract infection, Infect. Immun. 58 (1990) 1120-1123.

[12] P. Kosikowska, L. Berlicki, Urease inhibitors as potential drugs for gastric and urinary tract infections: a patent review, Expert Opin. Ther. Pat. 21 (2011) 945-957.

[13] S. Benini, M. Cianci, L. Mazzei, S. Ciurli, Fluoride inhibition of Sporosarcina pasteurii urease: structure and thermodynamics, J. Biol. Inorg. Chem. 19 (2014) 1243-1261.

[14] L. Mazzei, M. Cianci, S. Benini, L. Bertini, F. Musiani, S. Ciurli, Kinetic and structural studies reveal a unique binding mode of sulfite to the nickel center in urease, J. Inorg. Biochem. 154 (2016) 42-49. 
[15] S. Benini, W.R. Rypniewski, K.S. Wilson, S. Ciurli, S. Mangani, The complex of Bacillus pasteurii urease with $\beta$-mercaptoethanol from X-ray data at $1.65 \AA$ resolution, J. Biol. Inorg. Chem. 3 (1998) 268-273.

[16] L. Mazzei, M. Cianci, F. Musiani, S. Ciurli, Inactivation of urease by 1,4-benzoquinone: chemistry at the protein surface, Dalton Trans. 45 (2016) 5455-5459.

[17] J.M. Bremner, L.A. Douglas, Inhibition of urease activity in soils, Soil Biol. Biochem. 3 (1971) 297-307.

[18] R. Puupponen-Pimiä, A.M. Aura, K.M. Oksman-Caldentey, P. Myllärinen, M. Saarela,

T. Mattila-Sandholm, K. Poutanen, Development of functional ingredients for gut health, Trends Food Sci. Tech. 13 (2002) 3-11.

[19] E. Pastene, H. Speisky, A. Garcia, J. Moreno, M. Troncoso, G. Figueroa, In vitro and in vivo effects of apple peel polyphenols against Helicobacter pylori, J. Agric. Food Chem. 58 (2010) 7172-7179.

[20] M. Daglia, Polyphenols as antimicrobial agents, Curr. Opin. Biotechnol. 23 (2012) 174181.

[21] L. Paulo, M. Oleastro, E. Gallardo, J.A. Queiroz, F. Domingues, Anti-Helicobacter pylori and urease inhibitory activities of resveratrol and red wine, Food Res. Int. 44 (2011) 964-969.

[22] F. Cardona, C. Andrés-Lacueva, S. Tulipani, F.J. Tinahones, M.I. Queipo-Ortuño, Benefits of polyphenols on gut microbiota and implications in human health, J. Nutrit. Biochem. 24 (2013) 1415-1422.

[23] W. Kabsch, Xds, Acta Crystallogr. D Biol. Crystallogr. 66 (2010) 125-132.

[24] P. Evans, Scaling and assessment of data quality, Acta Crystallogr. D Biol. Crystallogr. $62(2006) 72-82$. 
[25] S. Benini, P. Kosikowska, M. Cianci, L. Mazzei, A.G. Vara, L. Berlicki, S. Ciurli, The crystal structure of Sporosarcina pasteurii urease in a complex with citrate provides new hints for inhibitor design, J. Biol. Inorg. Chem. 18 (2013) 391-399.

[26] G.N. Murshudov, A.A. Vagin, E.J. Dodson, Refinement of macromolecular structures by the maximum-likelihood method, Acta Crystallogr. D Biol. Crystallogr. 53 (1997) 240-255. [27] P. Emsley, K. Cowtan, Coot: model-building tools for molecular graphics, Acta Crystallogr. D Biol. Crystallogr. 60 (2004) 2126-2132.

[28] R.P. Joosten, F. Long, G.N. Murshudov, A. Perrakis, The PDB_REDO server for macromolecular structure model optimization, IUCrJ. 1 (2014) 213-220.

[29] E.F. Pettersen, T.D. Goddard, C.C. Huang, G.S. Couch, D.M. Greenblatt, E.C. Meng, T.E. Ferrin, UCSF Chimera - A visualization system for exploratory research and analysis, J. Comput. Chem. 25 (2004) 1605-1612.

[30] L. Schrodinger, 2015.

[31] F. Neese, The ORCA program system, Wiley Interdiscip. Rev. Comput. Mol. Sci. 2 (2012) 73-78.

[32] A.D. Becke, A new mixing of Hartree-Fock and local density-functional theories, J. Chem. Phys. 98 (1993) 1372-1377.

[33] C. Lee, W. Yang, R.G. Parr, Development of the Colle-Salvetti correlation-energy formula into a functional of the electron density, Phys. Rev. B 37 (1988) 785-789.

[34] M.J. Frisch, G.W. Trucks, H.B. Schlegel, G.E. Scuseria, M.A. Robb, J.R. Cheeseman, G. Scalmani, V. Barone, B. Mennucci, G.A. Petersson, H. Nakatsuji, M. Caricato, X. Li, H.P. Hratchian, A.F. Izmaylov, J. Bloino, G. Zheng, J.L. Sonnenberg, M. Hada, M. Ehara, K. Toyota, R. Fukuda, J. Hasegawa, M. Ishida, T. Nakajima, Y. Honda, O. Kitao, H. Nakai, T. Vreven, J.A. Montgomery Jr., J.E. Peralta, F. Ogliaro, M.J. Bearpark, J. Heyd, E.N. Brothers, K.N. Kudin, V.N. Staroverov, R. Kobayashi, J. Normand, K. Raghavachari, A.P. Rendell, J.C. Burant, S.S. Iyengar, J. Tomasi, M. Cossi, N. Rega, N.J. Millam, M. Klene, J.E. Knox, 
J.B. Cross, V. Bakken, C. Adamo, J. Jaramillo, R. Gomperts, R.E. Stratmann, O. Yazyev, A.J. Austin, R. Cammi, C. Pomelli, J.W. Ochterski, R.L. Martin, K. Morokuma, V.G. Zakrzewski, G.A. Voth, P. Salvador, J.J. Dannenberg, S. Dapprich, A.D. Daniels, Ö. Farkas, J.B. Foresman, J.V. Ortiz, J. Cioslowski, D.J. Fox, Gaussian, Inc., Wallingford, CT, USA, 2009. [35] M.J. Frisch, J.A. Pople, J.S. Binkley, Self-consistent molecular orbital methods 25. Supplementary functions for Gaussian basis sets, J. Chem. Phys. 80 (1984) 3265-3269. [36] T.H. Dunning, Gaussian basis sets for use in correlated molecular calculations. I. The atoms boron through neon and hydrogen, J. Chem. Phys. 90 (1989) 1007-1023.

[37] R.B. Silverman, Mechanism-based enzyme inactivators, Methods Enzymol 249 (1995) 240-283.

[38] H. Wagreich, J.M. Nelson, On the oxidation product of catechol when oxidized by means of tyrosinase, J. Biol. Chem. 115 (1936) 459-465.

[39] G.E.K. Branch, M.A. Joslyn, The kinetics of the auto-oxidation of catechol in the presence of several foreign substances, J. Am. Chem. Soc. 57 (1935) 2388-2394. [40] M.V. Vener, A.V. Odinokov, C. Wehmeyer, D. Sebastiani, The structure and IR signatures of the arginine-glutamate salt bridge. Insights from the classical MD simulations, J. Chem. Phys. 142 (2015) 215106.

[41] M. Kot, W. Zaborska, Irreversible inhibition of jack bean urease by pyrocatechol, J. Enzyme Inhib. Med. Chem. 18 (2003) 413-417.

[42] S. Benini, W.R. Rypniewski, K.S. Wilson, S. Miletti, S. Ciurli, S. Mangani, A new proposal for urease mechanism based on the crystal structures of the native and inhibited enzyme from Bacillus pasteurii: why urea hydrolysis costs two nickels, Structure 7 (1999) 205-216.

[43] P. Politzer, J.S. Murray, P. Lane, $\sigma$-Hole bonding and hydrogen bonding: Competitive interactions, Int. J. Quantum Chem. 107 (2007) 3046-3052. 
[44] X.M. Shen, G. Dryhurst, Iron- and manganese-catalyzed autoxidation of dopamine in the presence of L-cysteine: possible insights into iron- and manganese-mediated dopaminergic neurotoxicity, Chem. Res. Toxicol. 11 (1998) 824-837.

[45] N. Schweigert, A.J. Zehnder, R.I. Eggen, Chemical properties of catechols and their molecular modes of toxic action in cells, from microorganisms to mammals, Environ. Microbiol. 3 (2001) 81-91.

[46] R.F. Weiss, The solubility of nitrogen, oxygen and argon in water and seawater, DeepSea Research 17 (1970) 721-735.

[47] G. Lente, Deterministic kinetics in chemistry and systems biology, Springer International Publishing, 2015.

[48] Á. Balogh, G. Lente, J. Kalmár, I. Fábián, Reaction schemes that are easily confused with a reversible first-order reaction, Int. J. Chem. Kinet. 47 (2015) 773-782. 


\section{TABLES}

Table 1. Selected distances around the Ni(II) ions in native (PDB code 4CEU) and catecholbound SPU (PDB code 5G4H).

\begin{tabular}{|c|c|c|}
\hline $\mathrm{Ni}-\mathrm{L}$ distances $(\stackrel{\mathrm{A}}{)})$ & 4CEU & 5G4H \\
\hline $\mathrm{Ni}(1)-\alpha$ Lys $220 * \mathrm{O} \theta 1$ & 1.94 & 2.02 \\
\hline $\mathrm{Ni}(1)-\mathrm{O}(\mathrm{B})^{\mathrm{a}}$ & 2.08 & 1.90 \\
\hline $\mathrm{Ni}(1)-\mathrm{O}(1)$ & 2.24 & 2.21 \\
\hline $\mathrm{Ni}(1)-\alpha \mathrm{His} 249 \mathrm{~N} \delta$ & 2.03 & 2.04 \\
\hline $\mathrm{Ni}(1)-\alpha \mathrm{His} 275 \mathrm{~N} \varepsilon$ & 2.02 & 2.09 \\
\hline $\mathrm{Ni}(2)-\alpha \operatorname{Lys} 220 * \mathrm{O} \theta 2$ & 2.08 & 2.09 \\
\hline $\mathrm{Ni}(2)-\mathrm{O}(\mathrm{B})$ & 2.12 & 1.97 \\
\hline $\mathrm{Ni}(2)-\mathrm{O}(2)^{\mathrm{c}}$ & 2.07 & 2.17 \\
\hline $\mathrm{Ni}(2)-\alpha$ His $137 \mathrm{~N} \varepsilon$ & 2.11 & 2.10 \\
\hline $\mathrm{Ni}(2)-\alpha$ His139 $\mathrm{N} \varepsilon$ & 2.08 & 2.10 \\
\hline $\mathrm{Ni}(2)-\alpha A s p 363 \mathrm{O} \delta 1$ & 2.10 & 2.13 \\
\hline $\mathrm{Ni}(1) \cdots \mathrm{Ni}(2)$ & 3.67 & 3.57 \\
\hline $\mathrm{O}(1) \cdots \mathrm{O}(2)$ & 2.37 & 2.33 \\
\hline
\end{tabular}




\section{SCHEMES}

Scheme 1. Proposed reaction pathway for the SPU inactivation by OSQ leading to the formation of INT1, based on quanto-mechanical calculations.

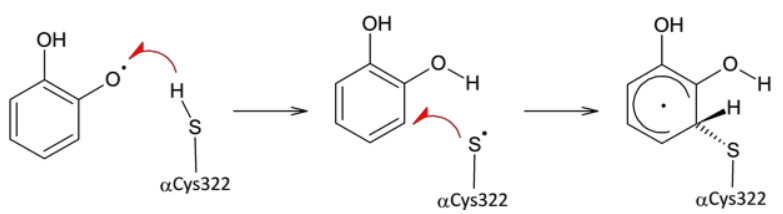

Scheme 2. Working hypothesis for the mechanism of inactivation of urease by catechol.

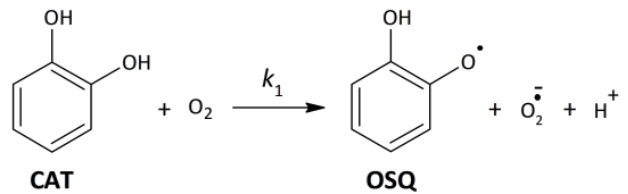<smiles>CC(C)CC1C(=O)C=CC(=O)C1Oc1ccc(Oc2ccccc2)c(O)c1</smiles>

CAT OBQ osa

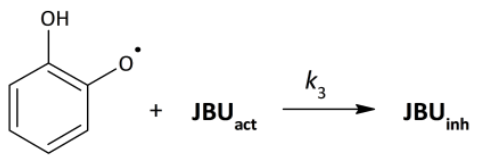

OSQ

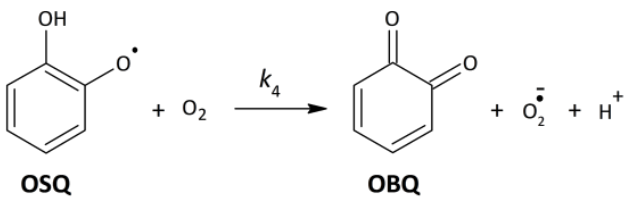




\section{FIGURE LEGENDS}

Figure 1. (A) Coordination environment of the Ni(II) ions in the active site of SPU (PDB code 4CEU [13]). Ribbons and longitudinal section of the open (B, PDB code 4CEU) and closed (C, PDB code 3UBP [42]) conformations of the flexible flap in SPU.

Figure 2. Plots of SPU (A) and JBU (B) residual activity $v s$. time of incubation at different catechol concentrations. In panel $\mathbf{B}$, the lines represent the result of the global fits of the data for JBU using Eq. 1 (see below).

Figure 3. Atomic model of the active site of catechol-inactivated SPU (PDB code 5G4H). In panel (A), the nickel-coordination environment is shown superimposed on the final $2 F_{\mathrm{o}}-F_{\mathrm{c}}$ electron density map contoured at $1.2 \sigma$; the map of the inhibitor is shown in yellow. In panel (B), the corresponding structural model is shown. H-bonds are shown as thin blue lines. Table S7 reports the corresponding numbers of the water molecules in the deposited PDB file.

Figure 4. Results of the relaxed surface scan computations and critical points identified along the reaction pathways between OSQ and MeSH. Atoms are colored accordingly to atom type. The inset reports the spin density calculated for the OSQ moiety. 


\section{FIGURES}

Figure 1.
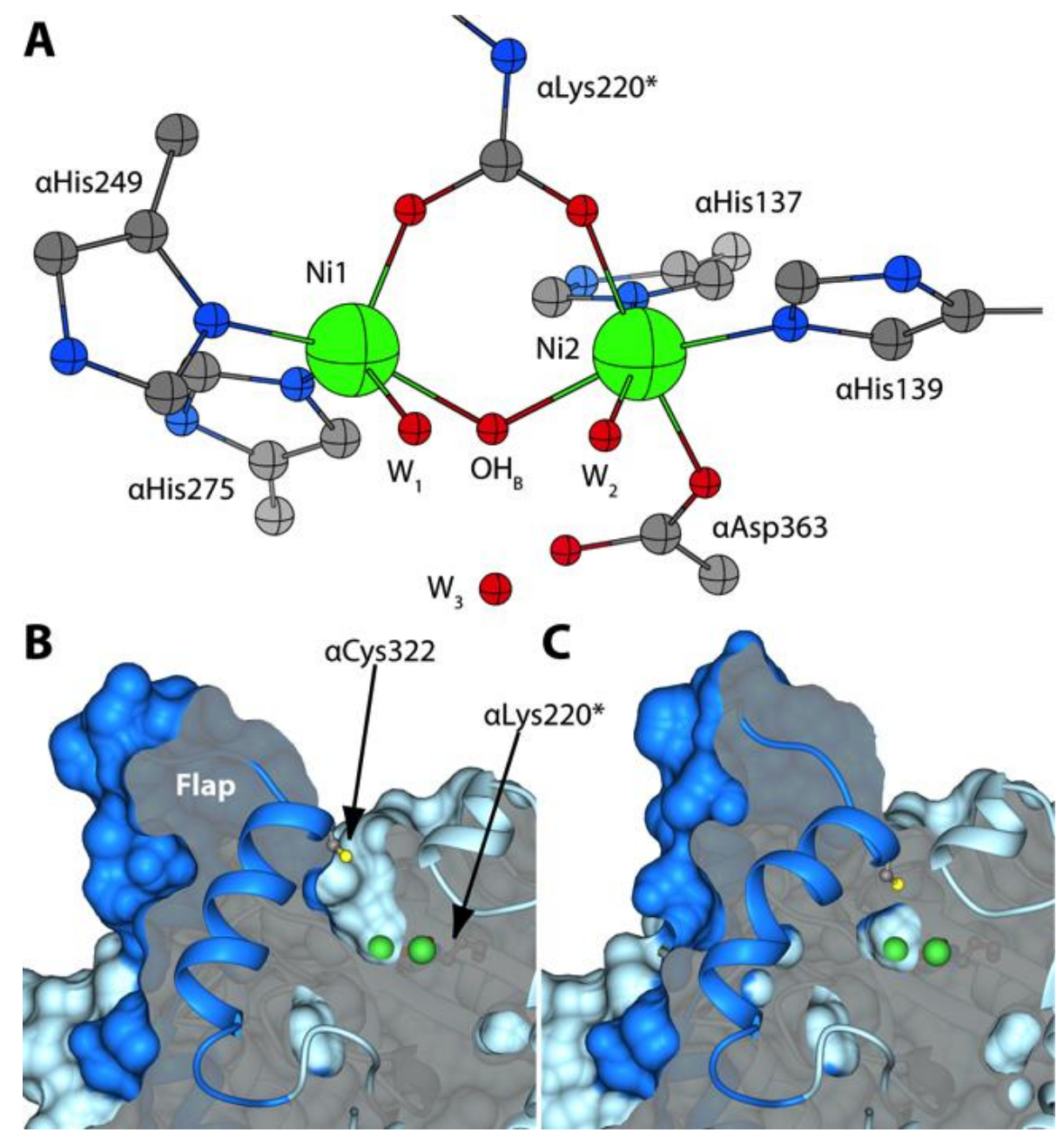
Figure 2.
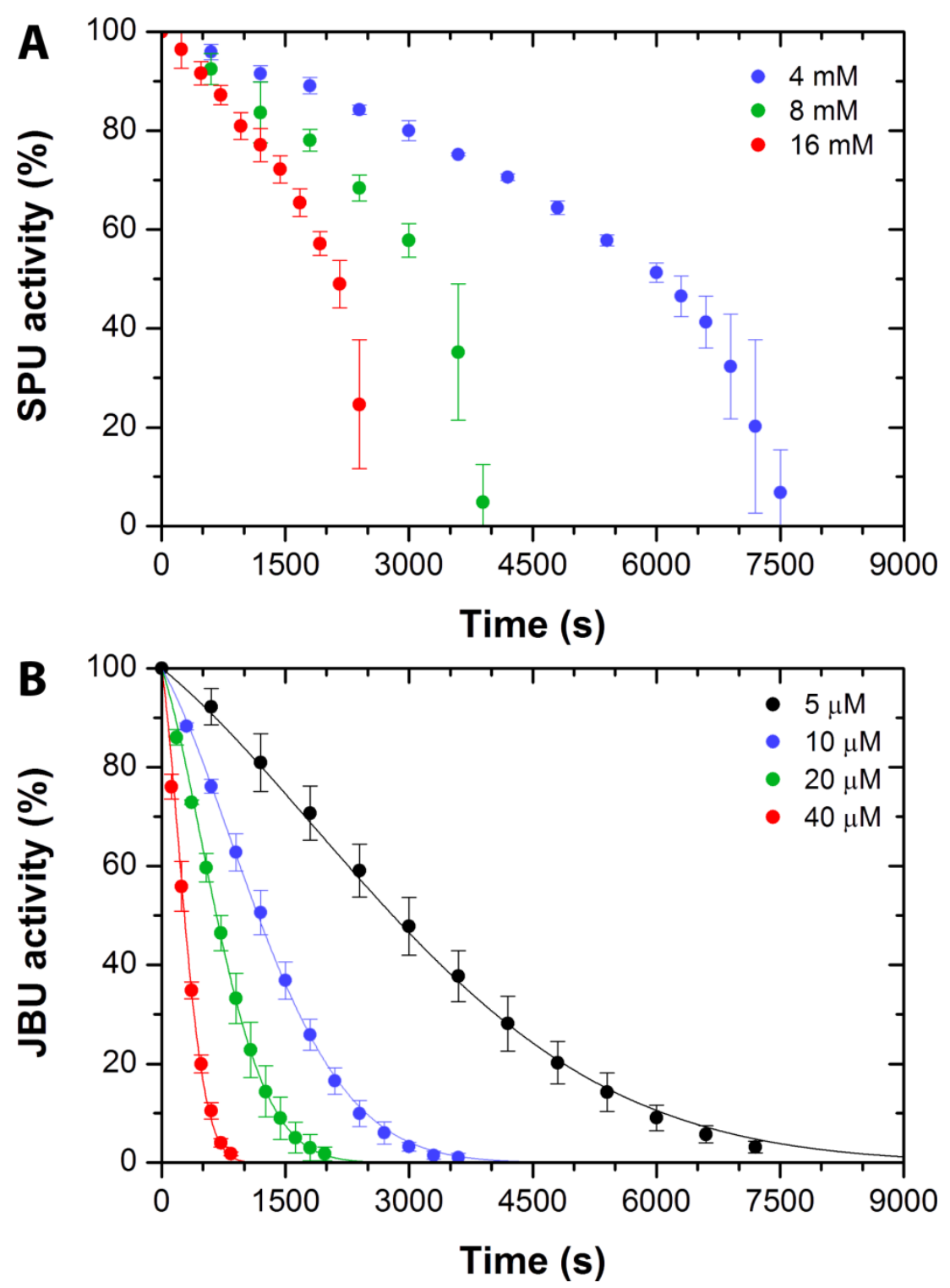


\section{Figure 3.}

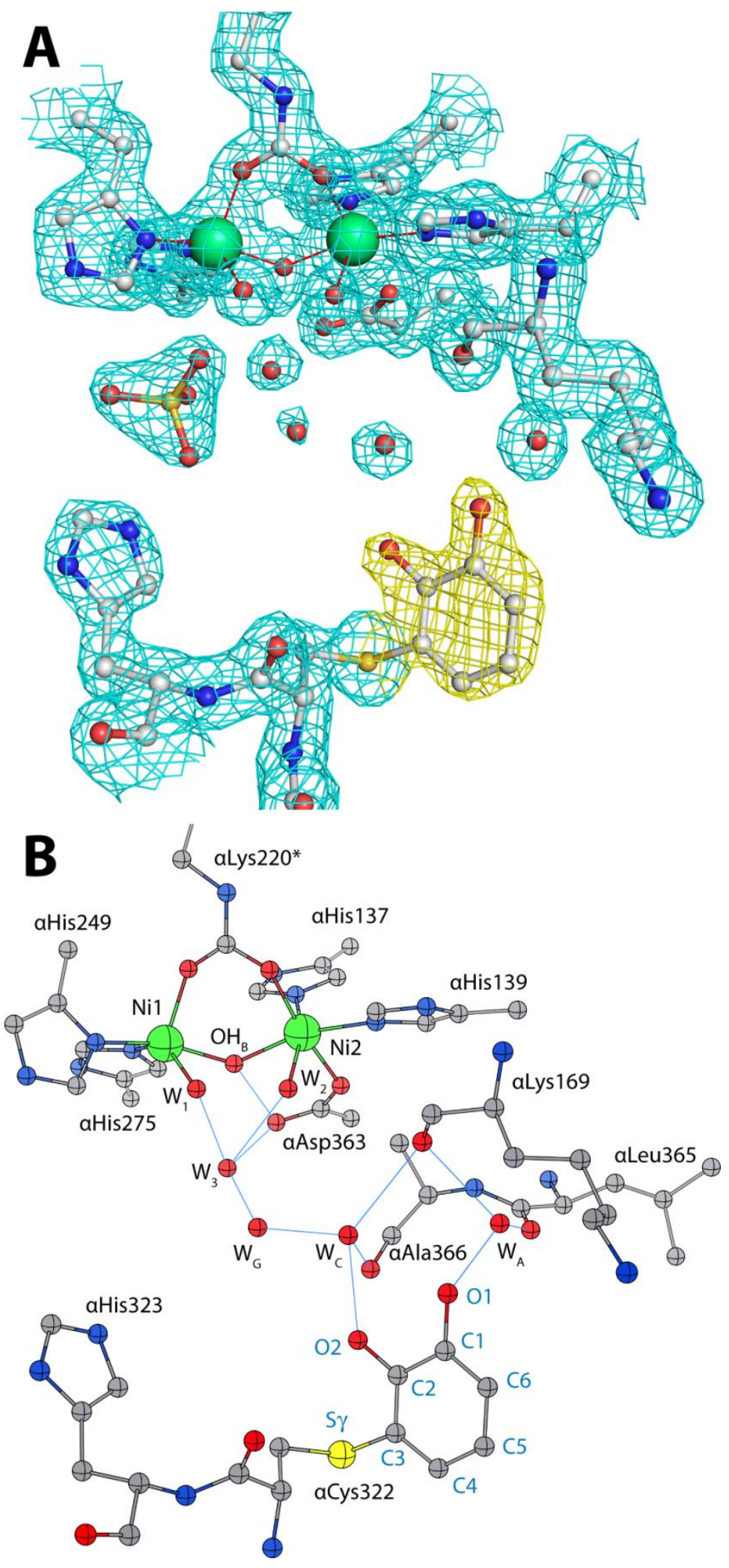


Figure 4.

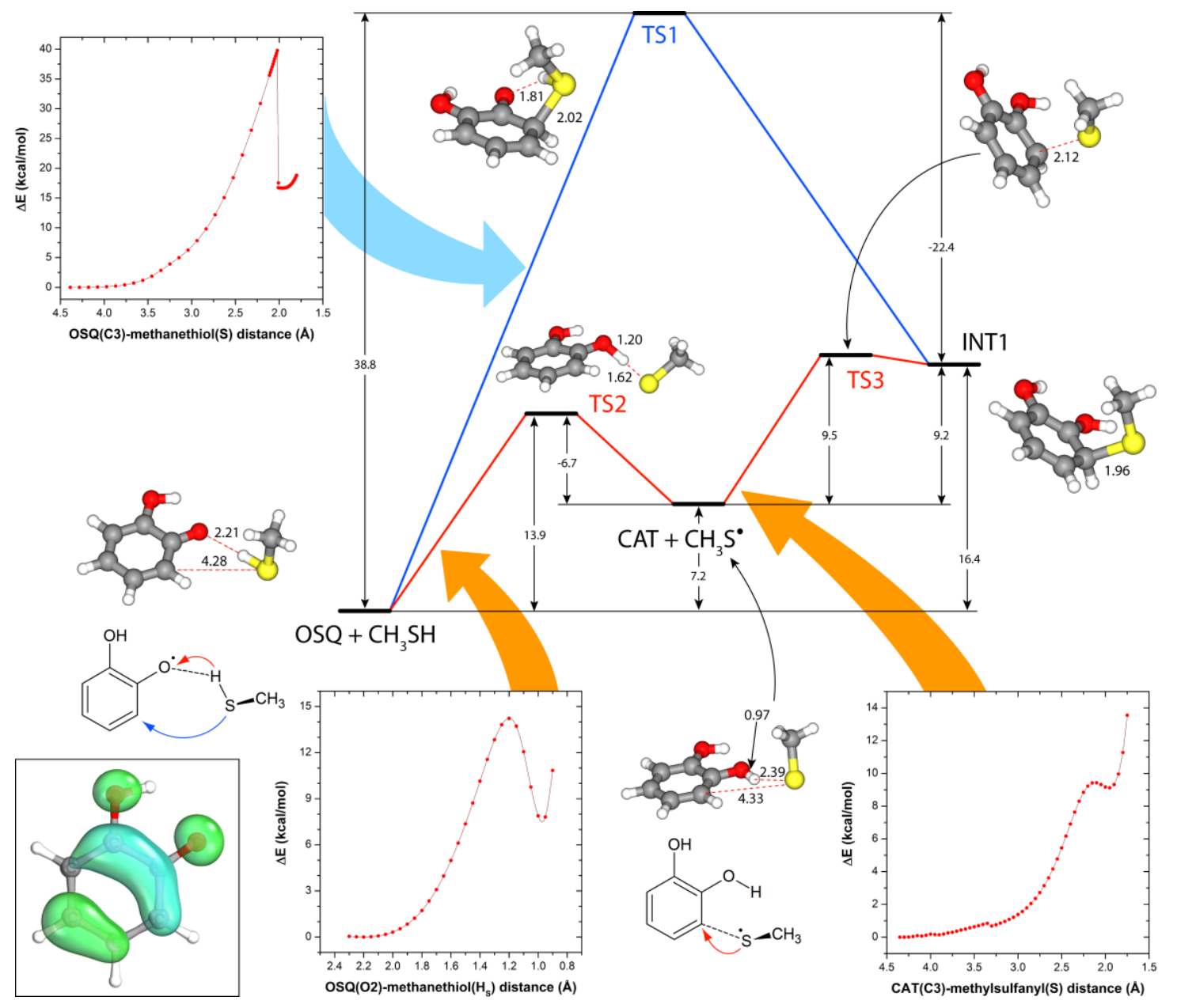


Supplementary Information for Publication Online
Click here to download Supplementary Informatior Click here to download Supplementary Information for Publication Online: Urease_catechol_JIB_rev_SI.pdf (n) (1) 